\title{
97. An Observation on the Brown-McCoy Radical
}

\author{
By F. SzÁsz
}

Mathematical Institute of Academy of Science, Budapest

(Comm. by K. KunUGI, M.J.A., July 12, 1961)

We wish to characterize in this note the Brown-McCoy radical $G(A)$ of an associative ring $A$, as a radical $(1,1,1,1)(A),(1,1,1,0)(A)$, $(1,1,0,1)(A)$ and $(1,2,1,1)(A)$, respectively, where $(k, l, m, n)(A)$ is a well-defined special $F$-radical of the ring $A$ in the sense of BrownMcCoy [3] for arbitrary nonnegative integers $k, l, m$ and $n$. The concept of a $(k, l, m, n)$-radicalring $A$ can be illustrated by the following elementary remarks. If the elements of $A$ form on the operation $a \circ b=a+b-a b(a, b \in A)$ a Neumann-regular semigroup (for instance in the case of a Jacobson-radicalring $A$, when $(A, 0)$ is a group), then $A$ is a $(k, 0,1,1)$-radicalring and a $(0, l, 1,1)$-radicalring at the same time for any integers $k, l \geqq 0$. Furthermore any $(k, l$, $m, n$ )-semisimple ring $A$ with minimum condition on twosided principal ideals is, as an $(A, A)$-doublemodule, completely reducible in a weak meaning, which generalizes the classical Wedderburn-Artin structure theorem also. (For the details of radicals, see [1], [2], [3].)

In this note the knowing of the results of Brown-McCoy [3] will be assumed for the reader. We denote the sum of all twosided principal ideals $\left(a^{(m)} \circ x \circ a^{(n)}-k \cdot a^{(l)}\right)$ by $(k, l, m, n)(a)$, where $a$ is a fixed element, $X$ a varying element of $A, a \circ b=a+b-a b, a^{(0)}=0, a^{(1)}=a$, $a^{(k+1)}=a^{(k)} \circ a$ and $k, l, m, n$ are nonnegative integers. An element $a \in A$ is called $(k, l, m, n)$-regular, if $a \in(k, l, m, n)(a)$. We call an element $a \in A$ strictly $(k, l, m, n)$-regular, if any element $b$ of the twosided principal ideal (a) generated by $a$ is $(k, l, m, n)$-regular. The set $(k, l, m, n)(A)$ of all strictly $(k, l, m, n)$-regular-elements of $A$ is called the $(k, l, m, n)$-radical of $A$. This is evidently a special $F$ radical of $A[3]$. The rings with $(k, l, m, n)$-radical $(0)$ are called $(k, l, m, n)$-semisimple. We call a subdirectly irreducible $(k, l, m, n)$ semisimple ring $A$ shortly: $(k, l, m, n)$-primitive. An element $a \neq 0$ with the condition $(k, l, m, n)(a)=0$ is called here a $(k, l, m, n)$ distinguished element of $A$. By [3] the $(k, l, m, n)$-radical of $A$ is the intersection of such ideals $\mathfrak{I}_{r}(\gamma \in \Gamma)$ of $A$, that the factorrings $A / \mathfrak{I}_{r}$ are $(k, l, m, n)$-primitive. $A /(k, l, m, n)(A)$ is $(k, l, m, n)$-semisimple, and a subdirect sum of $(k, l, m, n)$-primitive rings. By [3] a subdirectly irreducible ring $A$ is $(k, l, m, n)$-primitive if and only if the minimal ideal $\mathfrak{D} \neq 0$ of $A$ contains a $(k, l, m, n)$-distinguished element $d \neq 0$ playing the role of unity element in the case of radical 
$(1,1,1,1)(A)=G(A)$ of $A$.

Then holds the following

Theorem. An arbitrary $(k, l, m, n)$-primitive ring $P$ has no proper twosided ideals, and we have $\left(1-d^{(m)}\right) P\left(1-d^{(n)}\right)=0, d=k d \cdot d^{(l)}$, $k d^{(l)}=d^{(m+n)}$ for a $(k, l, m, n)$-distinguished element $d(\neq 0)$ of $P$. Furthermore $G(A)=(1,1,1,1)(A)=(1,1,1,0)(A)=(1,1,0,1)(A)=(1,2,1,1)$ $(A)$ are valid for the Brown-McCoy radical $G(A)$ of an arbitrary (associative) ring $A$.

Proof. If $P$ is $(k, l, m, n)$-primitive, then there exists [3] a $(k$, $l, m, n)$-distinguished element $d \neq 0$ in the minimal ideal $\mathbb{D} \neq 0$ of $P$. We have from $(k, l, m, n)(d)=0$ evidently $d^{(m)} \circ x \circ d^{(n)}=k \cdot d^{(l)}$ for any $x \in P$. In the special case $X=0$ follows $d^{(m+n)}=k d^{(l)}$ and thus in the case of arbitrary $x \in P$ is $X=d^{(m)} \cdot x+x d^{(n)}-d^{(m)} x d^{(n)} \in \mathbb{D}$ valid. Therefore one has $P=\mathfrak{D}$ for the $(k, l, m, n)$-primitive rings $P$, and thus $P$ cannot have proper twosided ideals. Obviously follows also $\left(1-d^{(m)}\right)$ $P\left(1-d^{(n)}\right)=0, d=d \cdot d^{(m+n)}$ and $d=k d \cdot d^{(l)}$ respectively. Let $A$ be now an arbitrary associative ring. Then $(1,1,1,1)(A)=G(A)$ will be proved by showing, that any $(1,1,1,1)$-primitive ring $P$ is a simple ring with unity element, and a similar fact holds for other special $k, l$, $m, n$ mentioned in the above theorem. In the four cases $k, l, m, n$ mentioned above, $k=1$, hence $d=d \cdot d^{(l)}$ and $d^{(l)}=d^{(m+n)}$. If $l=m=n$ $=1$, then one has $d^{2}=d$ for the $(k, l, m, n)$-distinguished element $d \neq 0$ of the $(k, l, m, n)$-primitive ring $P$. By $(1-d) P(1-d)=0$ follows $C=(1-d) P+P(1-d) P=0$, since $P$ is by $d^{2}=d \neq 0$ semi-simple in the sense of Jacobson, and the ideal $C$ is nilpotent. Thus $(1-d) P=0$, $P=d P\left(d^{2}=d\right)$ and similarly $P=P d$ too. Therefore one has $(1,1,1,1)$ $(A)=G(A)$. If $k=l=m=1$ and $n=0$, immediately follows

$$
(1,1,1,0)(\alpha)=\sum_{x \in A}\left(a \circ x \circ a^{(0)}-a\right)=\sum_{x \in A}(X-a x)=(1-a) A+A(1-a) A,
$$

and thus $(1,1,1,0)(A)=G(A)$ by the definition of the Brown-McCoy radical $G(A)$ of $A[3]$. The case $k=l=n=1$ and $m=0$ is totally similar to the previous case. If $k=m=n=1$ and $l=2$, then one has $d=d \cdot d^{(2)}$ and thus $d-2 d^{2}+d^{3}=0$. Then by $d=2 d^{2}-d^{3} \neq 0$ is surely $P^{2} \neq 0$, i.e. $P$ is semisimple in the sense of Jacobson by the want of proper ideals. By $(1-d) P(1-d)=0$ and $P^{2} \neq 0$ follows $C=(1-d) P$ $+P(1-d) P=0$, since $C$ is a nilpotent twosided ideal of $P$. This means $(1-d) P=0$ and $P=d P$. From $\left(d-d^{2}\right) P=(1-d) d P=0$ follows by $P^{2}$ $\neq 0$ evidently $d^{2}=d$, for a Jacobson-semisimple ring we have no annullator $\neq 0$. Therefore $d$ is a left unity element of $P(=d P)$, and similarly one has $P=P d$ also, which proves the theorem.

Remarks. 1) Any $(k, l, m, n)$-semisimple ring with minimum condition on twosided principal ideals is the discrete direct sum of $(k, l, m, n)$-primitive rings (see for these rings the above theorem), and conversely. 
2) If the elements of $A$ form with the operation $a \circ b=a+b-a b$ a Neumann-regular semigroup, then $A$ is a $(k, 0,1,1)$-radicalring and a $(0, l, 1,1)$-radicalring too.

3) It can be proved $A=(0,0,0,0)(A)=(k, 0,0,1)(A)=(0, l, 0,1)(A)$ $=(k, 0,1,0)(A)=(0, l, 1,0)(A)=(2,1,1,0)(A)=(2,1,0,1)(A)=(2,1,1,1)$ $(A)$.

For instance, if $P$ is a $(2,1,1,1)$-primitive ring, then holds $d^{(2)}=2 d^{(1)}$ and $(1-d) P(1-d)=0$, consequently $2 d-d^{2}=2 d, d^{2}=0$ and $0 \neq d=d$ $-2 d^{2}+d^{3}=(1-d) d(1-d) \in(1-d) P(1-d)=0$, which is a contradiction. Therefore $P=0$ and $(2,1,1,1)(A)=A$.

4) Any $(k, 0,1,1)$-primitive ring $P$ and any $(0, l, 1,1)$-primitive ring $P$ are simple rings with unity element and with the condition $2 P=P \neq 0$.

5) Any $(3,1,1,1)$-primitive ring, any $(3,1,1,0)$-primitive ring and any $(3,1,0,1)$-primitive ring $P$ are simple rings with unity element and with the condition $2 P=0$. Therefore for example a $(3,1$, $1,1)$-primitive ring $P \neq 0$ cannot be for instance a $(0, l, 1,1)$-primitive ring.

6) We have seen $(1,2,1,1)(A)=G(A)$. Then holds $(1,2,1,1)(a)$ $=((1-a) A(1-a))=(1-a) A(1-a)+A(1-a) A(1-a)+(1-a) A(1-a) A$ $+A(1-a) A(1-a) A \supseteq W(a)=A(1-a) A(1-a) A$. The following $W$-regularity: $b \in W(b)$ determines a special $F$-radical $W(A)$ of $A$. If $P$ is a $W$-primitive ring i.e. a $W$-semisimple and subdirectly irreducible ring, and if $P^{3} \neq 0$, then $P$ is a simple ring with unity element. If $P$ is a $W$-primitive ring and if $P^{2}=0$, then the additive group $P^{+}$ is isomorphic to a group $C\left(p^{k}\right)$, where $1 \leqq k \leqq \infty$. If finally $P^{2} \neq 0$ but $P^{3}=0$, and $P$ is a $W$-primitive ring, then we have $P \mathfrak{D}=\mathfrak{D} P=0$ for the minimal ideal $D$ of $P$ and $\left(P^{2}\right)^{+} \cong C\left(p^{k}\right)$ holds $(1 \leqq k \leqq \infty)$. For example $A=\left\{a_{1}, a_{2}, \cdots ; b_{1}, b_{2}, \cdots\right\}$ with $a_{i}^{2}-b_{i}=p a_{1}=b_{i}-p b_{i+1}=a_{i} a_{j}=a_{i}^{3}$ $=0$ is a $W$-primitive ring with $A^{3}=0$ and $A^{2} \neq 0,\left(A^{2}\right)^{+} \cong C\left(p^{\infty}\right)(i \neq j)$.

7) Let $A$ be an associative ring, $M$ a right $A$-module and $\mathfrak{M}$ an arbitrary cardinal number. An $A$-submodule $K$ of $M$ is called $\mathfrak{M}$ homoperfect, if the following conditions are satisfied:

I) $M A+K=M$;

II) $M / K$ is a completely reducible $A$-module of dimension $<M$;

III) $M / K$ has no proper $A$-submodule, which is invariant for all $A$-endomorphism of $M / K$;

IV) if $\varphi$ is an $A$-homomorphism of $M / L$ onto $M / K$ for an $A$ submodule $L$ with the conditions I), II) and III), then $\varphi$ is an isomorphism.

Let $\Re_{m}(M)$ be now itself $M$, if $M$ has no proper $M$-homoperfect submodules. If there exist in $M$ proper $M$-homoperfect submodules $K_{r}(\gamma \in \Gamma)$, then we define $\Re_{m}(M)=\bigcap_{r} K_{r}$. In the case of $1 \in A$, a unitary 
$A$-module $M$ and $\mathfrak{M}=2 ; \mathfrak{\imath}_{m}(M)$ is the Bourbaki-radical of $M$ [2], and in the case $M=2$ and arbitrary $A$ we obtain the Kertész-radical of $M[5]$. We have proved solving in [6] a problem of Dr. A. Kertész [5] that the Jacobson-radical $\widetilde{\digamma}(A)$ of $A$ must not coincide with the radical $\Re_{2}(A)$ of the right $A$-module $A$, if the power $|A|$ of $A$ is no quadratfree finite cardinal number. We have generally only $\mathfrak{R}_{2}(A) \subseteq \Im(A)$. If in the ring $A$ with left unity element holds the minimum condition on principal right ideals [7] and $\mathfrak{M}=\mathbb{E}_{0}$, then one has evidently $\mathfrak{R}_{y_{0}}(A) \subseteq G(A)$ for the above radical $\mathfrak{R}_{m}(A)$ of the right $A$-module $A$ and the Brown-McCoy radical $G(A)$ of $A$ * $^{*}$ Now we arise the following

Problem. What is a necessary and sufficient condition concerning $A$ for the validity of $\mathfrak{R}_{s_{0}}(A)=G(A)$ ? (Solve a similar problem of $A$. Kertész on $\mathfrak{R}_{2}(A)$ and $\dddot{\mho}(A)$ too!)

\section{References}

[1] W. A. Andrunakiewitsh: Semiradical rings (in Russian), Isvestiya Acad. Nauk. S.S.S.R. Ser. Math., 12, 129-178 (1948).

[2] N. Bourbaki: Éléments de Mathématiques, Algebre, Ch. VIII. Modules et Anneaux Semisimples, Hermann, Paris (1958).

[3] B. Brown and N. H. McCoy: Radicals and subdirect sums, Amer. Jour. Math., 69, 46-58 (1947).

[4] N. Jacobson: Structure of Rings, Providence (1956).

[5] A. Kertész: Investigations in the theory of operator-modules, III, Magyar Tud. Akad. III. Oszt. Közl., (in Hungarian), 9, 105-120 (1959).

[6] F. Szász: On the Kertész-radical of the operator-modules, Magyar Tud. Akad. III. Oszt. Közl., (in Hungarian), 10, 35-38 (1960).

[7] F. Szász: Über Ringe mit Minimalbedingung für Hauptrechtsideale, I, Publ. Math., Debrecen, 7, 54-64 (1960); II, Acta Math. Acad. Sci., Hung., 12 (in press).

*) It may be remarked that the theory of $F$-radicales can be formulated for $A$ modules too, where $F$ is a well-defined mapping of any $A$-module $M$ onto a set of submodules $F(m)$ of $M(m \in M, F(m) \subseteq M)$ with the condition $F(m) \varphi=F(m \varphi)$ for any $A$ homomorphism $\varphi$ of $M$. Then $m \in M$ is $F$-regular in the case $m \in F(m)$. Then the $F$ radical of $M$ is the set $[m ; m \in M, n \in F(n), n \in\{m\}]$. 